\title{
Concordancia en la medición testicular de varones adolescentes con 3 métodos de orquidometría
} Concordance of testicular measurement in male adolescents with three methods of orchidometry

\author{
Dr. Gonzalo Agüero y Dr. Enrique Berner ${ }^{a}$
}

\section{RESUMEN}

Introducción. El orquidómetro de Prader es el método estándar para medir el volumen testicular (VT) en niños y adolescentes.

Objetivo. Evaluar la concordancia en la estimación del VT y del inicio puberal con las técnicas de orquidometría dePrader, Chipkevitch y Sotos.

Métodos. Diseño descriptivo transversal realizado en varones de entre 9 y 20 años. Se midió el VT (ml) en cada adolescente con las técnicas de Prader (método de referencia), Chipkevitch (modelo gráfico) y Sotos (medición de ancho testicular con regla plástica y fórmula equivalente a ecuación elipsoide). Se excluyeron varones con patología urogenitaly enfermedades que afectan el crecimiento testicular. Para la concordancia entre métodos, se utilizó kappa para el inicio puberal, y coeficiente de correlación intraclase (CCI) y gráficos de Bland-Altman (GBA) para el VT.

Resultados. Se incluyeron 377 varones sanos. Para la concordancia en VT (ml), la comparación Prader-Chipkevitch obtuvo CCI: 0,994 y $\mathrm{p}<0,001$; y de CCI; 0,312 y $\mathrm{p}<0,001$ para la de Prader-Sotos. En los GBA se halló una media de las diferencias cercana a $0 \mathrm{ml}$ en la comparación Prader-Chipkevitch y cercana a $8 \mathrm{ml}$ en la de Prader-Sotos. El acuerdo en el inicio puberal obtuvo un valor de kappa 0,93 en la comparación

a. Servicio de Adolescencia, Hospital de Agudos Dr. Cosme Argerich, Ciudad Autónoma de Buenos Aires, Argentina.

Correspondencia: Dr. Gonzalo Agüero: aguerogonzalo@gmail. com

\section{Financiamiento: \\ Beca SAP de \\ investigación, \\ período 2018.}

Conflicto de intereses:

Ninguno que declarar.

Recibido: 31-10-2020

Aceptado: 8-2-2021

Cómo citar: Agüero G, Berner E. Concordancia en la medición testicular de varones adolescentes con 3 métodos de orquidometría. Arch Argent Pediatr 2021;119(4):251-258.

\section{INTRODUCCIÓN}

La medición del volumen testicular (VT) en niños y adolescentes es un indicador clínico que permite evaluar el desarrollo puberal y el componente testicular de enfermedades urogenitales, como el varicocele, y sistémicas sobre la función gonadal. ${ }^{1,2}$ Distintas sociedades científicas recomiendan realizar un examen genital anual en niños y varones adolescentes ${ }^{3,4}$ mediante la determinación de los estadios de Tanner y la estimación del VT con el orquidómetro de Prader. Este orquidómetro es el método de referencia en orquidometría clínica y en la detección del inicio puberal masculino. ${ }^{5,6}$

La concordancia es el grado en que dos o más observadores, métodos o técnicas están de acuerdo sobre el mismo fenómeno medido. Adquiere relevancia cuando se desea conocer si es posible obtener resultados equivalentes con un método o instrumento diferente al de uso habitual; de tal manera que, eventualmente, uno y otro puedan ser remplazados o intercambiados por cuestiones de facilidad de uso, costo y seguridad, entre otras. ${ }^{7}$

Nuestro objetivo fue evaluar la concordancia en la estimación del VT y del inicio puberal con los métodos de orquidometría de Prader, Chipkevitch $^{2}$ y Sotos ${ }^{1}$, con el primero como método de referencia.

\section{POBLACIÓN Y MÉTODOS}

Diseño: Estudio descriptivo transversal realizado en un hospital público entre el 1 de junio de 2018 y 
FIGURA 1. Medición del volumen testicular con tres técnicas de orquidometría

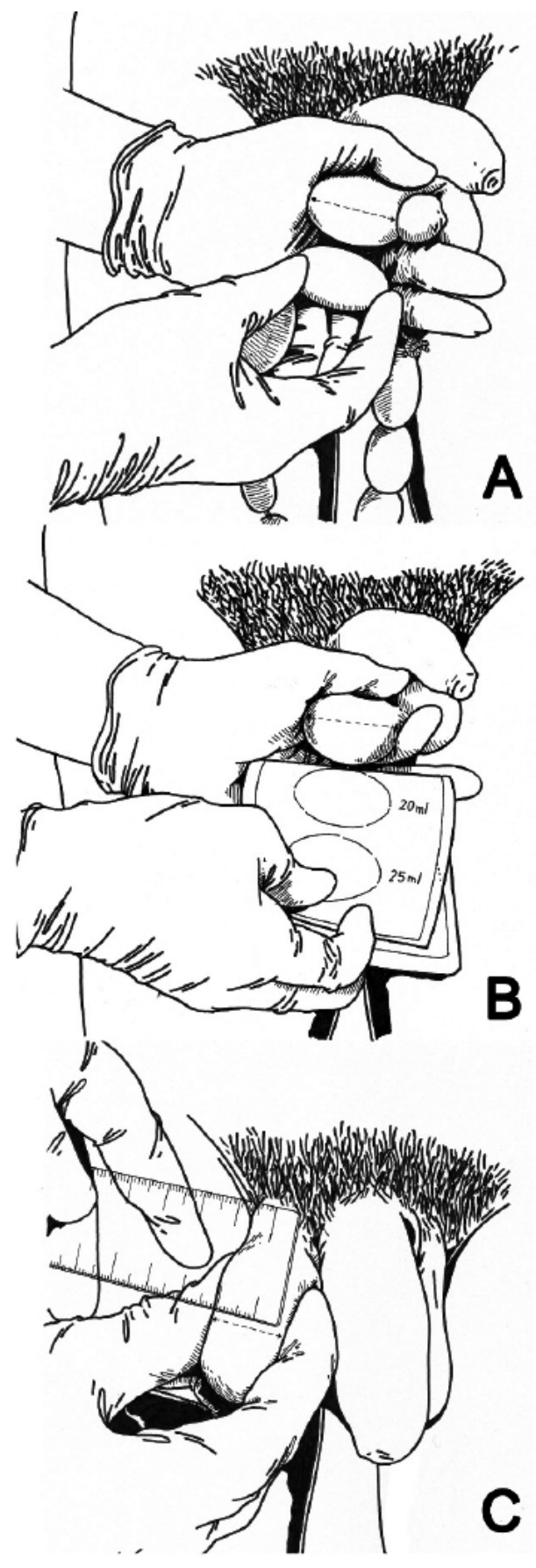

A: orquidómetro de Prader; B: método gráfico de Chipkevitch. Las técnicas A y B permiten comparar el eje mayor o largo testicular; por lo tanto, las cuentas del orquidómetro o la imagen de la tarjeta deben estar paralelas al eje mayor del testículo y lo más cerca posible de este; C: método de Sotos; esta técnica permite medir el ancho testicular y se recomienda realizar la medición visual desde 30 a $40 \mathrm{~cm}$ de distancia para neutralizar el paralaje. Fuente: los gráficos son autoría del Dr. Gonzalo Agüero. el 28 de febrero de 2020.

Población: Varones adolescentes.

Criterios de inclusión: varones argentinos de 9 a 20 años, sin antecedentes relevantes, que concurrieron a controles de salud.

Criterios de exclusión: alteraciones puberales, hipogonadismo, anomalías urogenitales congénitas, patología escrotal, enfermedades crónicas que afectan el crecimiento testicular, discapacidad intelectual, enfermedad aguda en curso y negativa a participar del estudio. Si algún adolescente cumplió con los criterios de inclusión, pero en el examen genital se detectó alguna de las patologías citadas, entonces fue eliminado del estudio.

\section{Variables}

Estadios de Tanner. ${ }^{8-10}$ Clasifica el desarrollo puberal en cinco estadios, según los cambios morfológicos de los genitales externos $(\mathrm{G})$ y vello púbico (VP). El estadio 1 se considera prepuberal; los estadios 2-4, pubertad en curso; y el estadio 5, desarrollo completo.

Volumen testicular (ml). Se midió el volumen de ambos testículos con tres técnicas orquidométricas. El orquidómetro de Prader se utiliza para medir por palpación comparativa entre el testículo y un ovoide de volumen conocido. ${ }^{11,12}$ Es el orquidómetro más utilizado para medir el volumen testicular, complementa la estadificación de Tanner y es de particular utilidad para discriminar el desarrollo prepuberal del peripuberal en adolescente tempranos. ${ }^{5,6}$ El método visual de Chipkevitch ${ }^{2,13}$ propone la comparación entre el testículo y una tarjeta con seis óvalos de proporciones conocidas. Por último, con el método de Sotos ${ }^{1,14}$ se realiza la medición del ancho testicular aplicado a una variante de la fórmula elipsoide que coincide con la medición ecográfica (Figura 1 y Tabla 1). Se considera que la ecografía permite estimar el VT con mayor precisión, aunque es un método poco práctico para la atención diaria de niños y adolescentes; por lo tanto, su uso se reserva para evaluación de problemas urogenitales. ${ }^{1,14}$

Inicio puberal. La pubertad masculina comienza al alcanzar un $\mathrm{VT} \geq 4 \mathrm{ml}$ en la orquidometría de Prader; este valor corresponde a un volumen ecográfico $\geq 1,4 \mathrm{ml} .{ }^{15}$ Dado que sus autores no describieron un valor de corte para el inicio puberal, se asume un $\mathrm{VT} \geq 1,4 \mathrm{ml}$ en el caso de Sotos (su método coincide con la ecografía) y $\geq 3,5 \mathrm{ml}$ en el caso de Chipkevitch (valor más próximo al indicado por orquidometría de Prader, 
ver Tabla 1). El inicio puberal se mide en términos de sí/no.

Muestreo. Se estimó el intervalo de confianza (IC) para una media, sobre la asunción de que la mayor variabilidad en el VT obtenido por orquidometría de Prader es a los 14 años (media: $15 \mathrm{ml}$, desviación estándar [DE] $7 \mathrm{ml}),{ }^{16} \mathrm{con}$ un IC $95 \%$ y una precisión de $2 \mathrm{ml}$; se requieren 50 adolescentes por cada estadio de Tanner (250 varones y 500 testículos). Los participantes se incluyeron de manera secuencial por un muestreo aleatorio simple, limitado por el número de consultas diarias realizadas por varones.
Procedimientos. El investigador principal evaluó a todos los participantes en la siguiente secuencia: estadificación de Tanner; palpación del contenido escrotal para excluir patología testicular, del cordón espermático o del epidídimo; y medición comenzando por el testículo derecho con las técnicas en el siguiente orden: Chipkevitch, Sotos y Prader.

Control de sesgos. Para evitar la influencia de factores urogenitales o enfermedades crónicas, se evaluaron solo adolescentes saludables, sin antecedentes clínicos relevantes.

Para obtener un VT confiable, todas las

TABla 1. Características de 3 técnicas de orquidometría clínica

\begin{tabular}{|c|c|c|c|}
\hline Caraterística & Orquidómetro de Prader & Método visual de Chipkevitch & Método de Sotos \\
\hline Instrumento & $\begin{array}{l}\text { Ovoides de volumen } \\
\text { conocido unidos por } \\
\text { una cuerda. }\end{array}$ & $\begin{array}{l}\text { Modelo gráfico o tarjeta con } \\
\text { seis óvalos de dimensiones } \\
\text { conocidas. }\end{array}$ & $\begin{array}{l}\text { Regla plástica transparente y } \\
\text { una variante de la fórmula de } \\
\text { ecuación elipsoide. }\end{array}$ \\
\hline Justificación del método & \multicolumn{2}{|c|}{$\begin{array}{l}\text { Se asume que el testículo es un elipsoide rotacional. } \\
\text { El VT se obtuvo a partir del ancho y largo testicular } \\
\text { (medidos con calibre o regla), y se aplicó la ecuación elipsoide: } \\
0,52 \times \mathrm{W}^{2} \times \mathrm{L} \\
\text { donde } \mathrm{W}=\text { ancho testicular y } \mathrm{L}=\text { longitud testicular. En el } \\
\text { cálculo se incluye la piel escrotal y otros tejidos adyacentes, } \\
\text { por esto ambos métodos sobrestiman el VT. }\end{array}$} & $\begin{array}{l}\text { Obtiene un VT similar al valor } \\
\text { ecográfico, al eliminar de su } \\
\text { cálculo los tejidos adyacentes } \\
\text { del testículo. Utiliza la } \\
\text { ecuación elipsoide: } \\
(\mathrm{W} \text {-ss })^{3} \times 0,88 \\
\text { donde } \mathrm{W}=\text { ancho testicular y } \\
\text { ss = doble piel escrotal, que } \\
\text { varía según el estadio de } \\
\text { Tanner }(\mathrm{G} 1, \mathrm{G} 2 \text { y G3 } \sim 1,5 \mathrm{~mm} \text {; } \\
\text { G4 y G5 } \sim \mathrm{mm}) \text {. }\end{array}$ \\
\hline Técnica (orquidometría) & $\begin{array}{l}\text { Se toma el testículo con una } \\
\text { mano; con la otra se toma el } \\
\text { orquidómetro y se compara } \\
\text { con la cuenta más parecida } \\
\text { en volumen o en eje mayor. }\end{array}$ & $\begin{array}{l}\text { Se toma testículo con una mano; } \\
\text { con la otra se toma la tarjeta y } \\
\text { se compara el eje mayor del } \\
\text { testículo con el eje mayor del } \\
\text { dibujo que más se aproxime. }\end{array}$ & $\begin{array}{l}\text { - Se determina el estadio } \\
\text { genital de Tanner. } \\
\text { - Se toma el testículo entre } \\
\text { índice y pulgar y } \\
\text { se mide su ancho. } \\
\text { - Se resta la doble piel escrotal } \\
\text { al ancho testicular. } \\
\text { - Se utilizan los datos en la } \\
\text { ecuación elipsoide. }\end{array}$ \\
\hline Escala & $\begin{array}{l}\text { Cuentas de } 1,2,3,4,5,6,8 \\
10,12,15,20,25 \mathrm{ml} \text { y } \\
\text { valores intermedios entre } \\
\text { cuentas no consecutivas. }\end{array}$ & $\begin{array}{l}\text { Óvalos de } 2,5,10,15,20,25 \mathrm{ml} \\
\text { y sus valores intermedios. } \\
\text { Los VT }<2 \mathrm{ml} \text { se asumieron } \\
\text { como iguales a } 1 \mathrm{ml} .\end{array}$ & $\begin{array}{l}\text { Depende de la medición del } \\
\text { ancho testicular: dado que no } \\
\text { tiene límite máximo de VT, } \\
\text { puede medir con exactitud } \\
\text { testículos grandes. }\end{array}$ \\
\hline Intrapolación & \multicolumn{2}{|c|}{$\begin{array}{l}\text { Cuando el volumen de un testículo quedó entre dos cuentas } \\
\text { u óvalos, se tomó el valor intermedio. Por ejemplo: } \\
\text { entre } 15 \text { y } 20 \mathrm{ml} \text {, se tomó } 17,5 \mathrm{ml} \text {. }\end{array}$} & No aplica. \\
\hline Extrapolación & \multicolumn{2}{|c|}{$\begin{array}{l}\text { Cuando el VT fue }>25 \mathrm{ml} \text { se registró como de } 25 \mathrm{ml} \text {, } \\
\text { dado que no se puede extrapolar de manera confiable. }\end{array}$} & No aplica. \\
\hline Costo* & $\$ 1000-1300$ & $\begin{array}{l}\text { Impresión y plastificado } \\
\text { de la tarjeta \$100-120. }\end{array}$ & Regla $\$ 30-40$ \\
\hline
\end{tabular}

VT: volumen testicular.

${ }^{*}$ Costos expresados en pesos argentinos, estimados en agosto de 2020.

Fuente: elaborado a partir de datos obtenidos de las referencias 1, 2, 11-14. 
mediciones se realizaron con las siguientes condiciones: se utilizaron los mismos instrumentos en cada medición, se cuidó que el ambiente estuviese templado $\left(\geq 20^{\circ} \mathrm{C}\right)$ para evitar el reflejo cremasteriano, y se disminuyó al máximo posible la piel escrotal circundante sin comprimir ni deformar el testículo.

El análisis estadístico fue cegado para compensar la falta de ciego en las mediciones del VT realizadas por el investigador principal.

Para evaluar la reproductibilidad de los métodos en diferentes observadores, se estimó el acuerdo intra e interobservador en 13 participantes que aceptaron ser evaluados por un segundo observador, durante los primeros tres meses del estudio. La comparación interobservador se realizó entre el investigador principal (pediatra con 10 años de experiencia en atención de adolescentes) y una médica generalista con un año de experiencia en atención de adolescentes. Cada observador evaluó de forma ciega el estadio puberal y el VT en dos oportunidades, tal como se describe en la sección Procedimientos.

Procesamiento y análisis de datos. Las variables cuantitativas se describieron con medidas de distribución central, desviación estándar (DE) y rango intercuartílico. Dado que el VT no se ajustó a una distribución normal (prueba de Kolmogorov-Smirnov), para su correlación y comparación se utilizó el coeficiente de Pearson y prueba de rangos con signo de Wilcoxon para muestras relacionadas, respectivamente. La concordancia entre variables categóricas (inicio puberal) se comparó con el coeficiente KappaCohen; para las variables cuantitativas (VT en

FIGURA 2. Flujograma de los adolescentes en el estudio

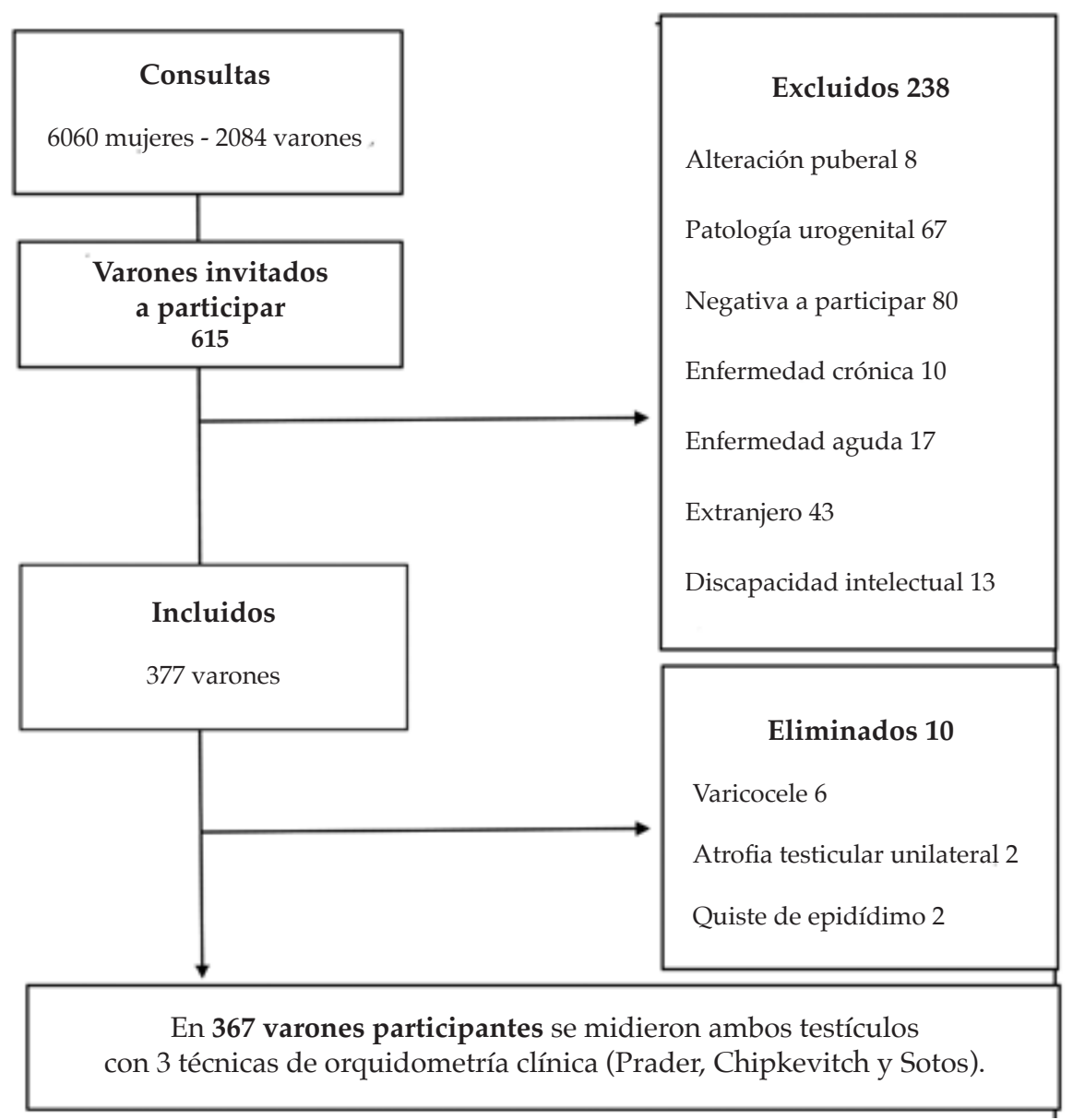


ml) se utilizaron el coeficiente de correlación intraclase (CCI) y los gráficos de Bland-Altman (GBA). El CCI permite medir concordancia entre dos o más valoraciones cuantitativas obtenidas con diferentes instrumentos o diferentes observadores; sus valores pueden oscilar entre 0 (ausencia de concordancia) y 1 (concordancia absoluta). Los GBA permiten comparar técnicas de medición sobre una misma variable cuantitativa y cuantificar la diferencia media entre ambos métodos y los límites de confianza, entre los cuales se incluyen el $95 \%$ de las diferencias entre una técnica de medición y la otra. Si ambos métodos concuerdan, la diferencia media se situará próxima a cero. Si se aleja de este valor, significa que los dos métodos producen resultados diferentes y uno de ellos subestima o sobrestima el valor del método de referencia.

Los datos fueron procesados con paquetes Epidat $4.2^{\circledR}$ (Galicia-OPS, 2016) y SPSS20 ${ }^{\circledR}$ (IBM, 2011).

TABLA 2. Concordancia intra e interobservador en la medición testicular con tres técnicas de orquidometría $(n=13)$

\begin{tabular}{lllll}
\hline \multirow{2}{*}{$\begin{array}{l}\text { Técnicas de } \\
\text { orquidometría }\end{array}$} & \multicolumn{3}{l}{ Concordancia } \\
\cline { 2 - 5 } & $\begin{array}{l}\text { Intraobservador: comparación de un observador } \\
\text { contra sí mismo en dos evaluaciones ciegas }\end{array}$ & $\begin{array}{l}\text { Interobservador: comparación entre dos } \\
\text { observadores en dos evaluaciones ciegas. }\end{array}$ \\
\cline { 2 - 5 } & Observadores & CCI (IC95 \%) & Evaluaciones & CCI (IC95 \%) \\
\hline Prader & Investigador principal (IP) & $0,970(0,928-0,987)$ & $1^{\circ}$ evaluación IP - SO & $0,998(0,995-0,999)$ \\
& Segundo observador (SO) & $0,873(0,736-0,941)$ & $2^{\circ}$ evaluación IP - SO & $0,888(0,767-0,948)$ \\
Chipkevitch & IP & $0,996(0,991-0,998)$ & $1^{\circ}$ evaluación IP - SO & $0,964(0,917-0,984)$ \\
& SO & $0,990(0,975-0,996)$ & $2^{\circ}$ evaluación IP - SO & $0,961(0,915-0,982)$ \\
Sotos & IP & $0,957(0,897-0,981)$ & $1^{\circ}$ evaluación IP - SO & $0,666(0,333-0,843)$ \\
& SO & $0,946(0,885-0,9756)$ & $2^{\circ}$ evaluación IP - SO & $0,768(0,451-0,900)$ \\
\hline
\end{tabular}

CCI: coeficiente de correlación intraclase, IC95\%: intervalo de confianza del $95 \%$.

El acuerdo o concordancia intra e interobservador con las técnicas de Prader y Chipkevitch fue excelente (cercano a 1). Para el método de Sotos el acuerdo intraobservador fue excelente, mientras que el acuerdo interobservador fue bueno en la primera evaluación y mejoró en la segunda evaluación. Todas las mediciones fueron estadísticamente significativas $(p<0,001)$.

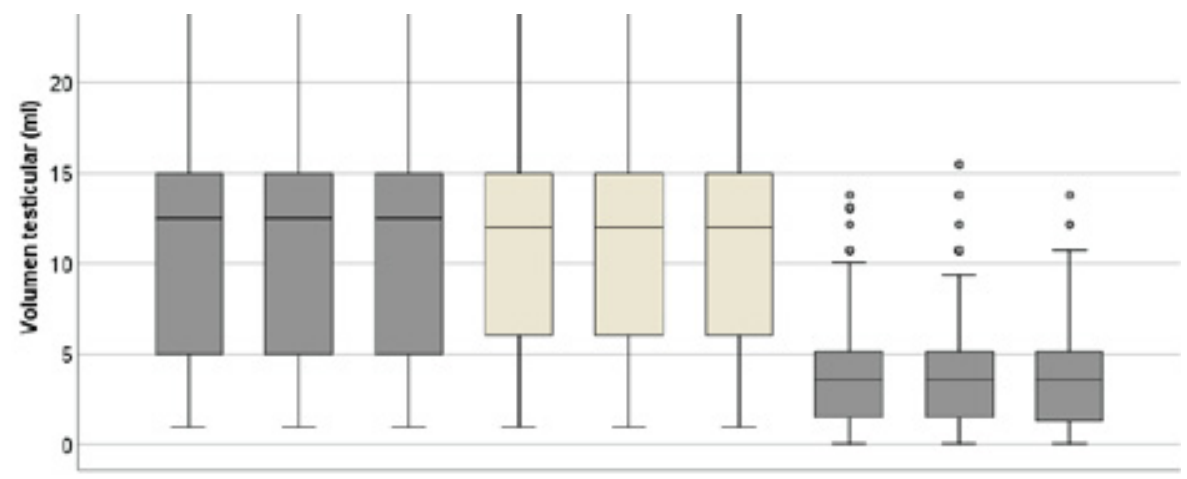

MVT: media de volumen testicular, TD: testículo derecho, TI: testículo izquierdo.

Estadística descriptiva. Orquidómetro de Chipkevitch: MVT 11,5 $\pm 7,9$ ml; mediana 12,5; rango intercuartílico 5-15 ml; mínimo 1 y máximo 25. Orquidómetro de Prader: MVT 11,7 \pm 6,9ml; mediana 12; rango intercuartílico 6-15 ml; mínimo 1 y máximo 25. Método de Sotos: MVT 3,9 3 ml; mediana 3,6; rango intercuartílico 2-5 ml; mínimo 0,4 y máximo 13,75. 


\section{Consideraciones éticas}

Se obtuvo la aprobación del Comité de Ética en Investigación del Hospital el 19 de marzo de 2018. Se solicitó el consentimiento informado a la familia y a los pacientes mayores de 14 años. La participación fue voluntaria, anónima y confidencial. Cada evaluación se realizó con respeto por la privacidad de los adolescentes.

\section{RESULTADOS}

La causa más frecuente de exclusión o eliminación por patología urogenital fue el varicocele, con un total de 24 casos. Los varones con patología urogenital fueron derivados para seguimiento médico.

Se evaluaron 367 varones con tres técnicas orquidométricas (Figura 2 y Tabla 2). La edad promedio fue de $13,8 \pm 2,5$ años.

Volumen testicular (VT). Con los tres métodos se observó que: 1) la distribución del VT no fue paramétrica (Figura 3); 2) existe una alta correlación entre los tamaños de los testículos derechos (TD) e izquierdos (TI) (coeficiente de
Pearson: 0,988 (Prader); 0,991 (Chipkevitch) y 0,967 (Sotos); p < 0,001); sin embargo, la comparación de medianas mostró que, en general, el TD fue mayor que el TI (prueba de rangos con signo de Wilcoxon para muestras relacionadas; p $<0,001) ; y 3)$ las técnicas de orquidometría tuvieron una alta correlación en la medición de los TD (coeficiente de Pearson: 0,993 (PraderChipkevitch) y 0,882 (Prader-Sotos); $\mathrm{p}<0,001$ ) y en los TI (coeficiente de Pearson: 0, 992 (PraderChipkevitch) y 0,889 (Prader-Sotos); p $<0,001$ ).

Con la orquidometría de Prader y Chipkevitch se identificaron diez adolescentes con VT $>25 \mathrm{ml}$, todos en estadio G5. De la misma manera, con la orquidometría de Sotos se detectaron ocho varones en estadio G5 con valores extremos en la distribución del VT.

Concordancia entre técnicas orquidométricas. La comparación Prader-Chipkevitch de TD obtuvo un CCI de 0,993; un IC95 \% de 0,9910,994; y un valor de $\mathrm{p}<0,001$. La comparación Prader-Chipkevitch de TI obtuvo un CCI de 0,992; IC95 \% 0,990-0,994; y p < 0,001. La comparación
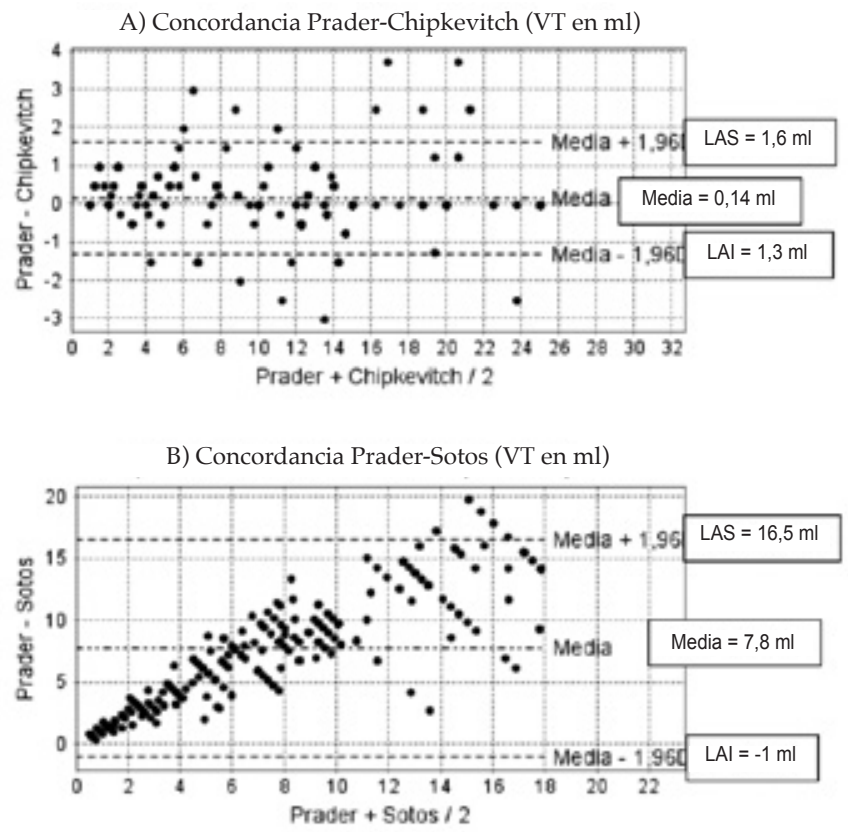

En el gráfico se muestra, a modo de ejemplo, el promedio entre testículo derecho e izquierdo, dado que sus valores individuales son similares.

VT: volumen testicular, LAS: límite de acuerdo superior, LAI: límite de acuerdo inferior.

Gráficos de Bland-Altman: la media de las diferencias y los límites de acuerdo sobre las diferencias, límite de acuerdo superior (LAS) e inferior (LAI), se muestran como líneas horizontales. $\mathrm{Y}=0$ es la línea de concordancia promedio perfecta. Si ambos métodos concuerdan, entonces la diferencia media se situará en cero o próxima a cero. Si se aleja de este valor, significa que los dos métodos producen resultados diferentes. La concordancia Chipkevitch-Sotos no se muestra en el gráfico; los valores fueron: media de las diferencias 8,2 ml (IC95 \% 7,6-8,7); DE 4,5 ml; LAI -0,6 ml; LAS 16,9 ml. 
Prader-Sotos de TD obtuvo un CCI de 0,320; IC95 \% -0,095-0,642; y p < 0,001; mientras que para TI obtuvo un CCI de 0,305; IC95 \% -0,0950,$624 ; \mathrm{y} p<0,001$. Los gráficos de Bland-Altman mostraron una media de las diferencias entre Prader y Chipkevitch cercana a cero tanto para TD como para TI; en cambio, la media de las diferencias entre Prader y Sotos estuvo cercana a $8 \mathrm{ml}$ para ambos testículos (Figura 4).

Inicio puberal. Con el orquidómetro de Prader como referencia, se evaluó el acuerdo entre métodos en la detección del inicio puberal. Para la comparación Prader-Chipkevitch el valor de kappa fue de 0,93; el IC95 \% de 0,87-0,98; y el valor de $\mathrm{p}<0,001$; para la comparación Prader-Sotos, los valores fueron de 0,$75 ; 0,67-0,83 \mathrm{y}<0,001$, respectivamente.

Acuerdo entre observadores. Cada observador realizó 26 evaluaciones de Tanner y 26 mediciones testiculares con tres técnicas orquidométricas en 13 adolescentes. El acuerdo intra e interobservador para clasificar el VP fue excelente (kappa 1; p <0,001), del mismo modo que el acuerdo intra e interobservador para clasificar el estadio G (kappa 1 y 0,887 , respectivamente; $p<0,001)$.

Orquidometría. Los resultados del CCI se muestran en la Tabla 2.

\section{DISCUSIÓN}

El crecimiento testicular puberal es un indicador indirecto de la función gonadal en niños y adolescentes, pues refleja el desarrollo de los túbulos seminíferos. ${ }^{16,17}$ La disponibilidad de instrumentos de orquidometría, sencillos y confiables, podría mejorar la evaluación del desarrollo puberal y de diferentes enfermedades que afectan el crecimiento testicular en niños y adolescentes. En este trabajo, se comparó la concordancia entre tres métodos de orquidometría (Prader, Chipkevitch y Sotos), con el de Prader como método de referencia.

El método gráfico de Chipkevitch mostró una muy buena correlación y concordancia con la orquidometría de Prader en la estimación del VT y del inicio puberal. Según el conocimiento de los autores, este el primer estudio en evaluar el método visual de Chipkevitch luego de su descripción original. ${ }^{2}$

En nuestra población, el rango del VT obtenido mediante el método de Sotos fue de 0,4 a 13,75 ml, similar al descripto por su autor $(0,5 \mathrm{a} 17,32 \mathrm{ml}))^{1,14}$ De manera coincidente, Koskenniemi et al., describen que un VT obtenido por ecografía es aproximadamente de 0,6 $\mathrm{ml}$ en la prepubertad y de $13 \mathrm{ml}$ en la pospubertad. ${ }^{17}$ En comparación con la orquidometría de Prader, la técnica de Sotos tuvo baja concordancia en la estimación del VT, pero buena en la detección del inicio puberal. La orquidometría de Prader tiene buena correlación con la ecografía, pero no de concordancia ${ }^{15-17}$ (la orquidometría de Prader sobrestima el VT respecto a la ecografía). El método de Sotos fue desarrollado para superar este obstáculo y, por ello, es esperable que, al igual que la ecografía, tenga buena correlación, pero no concordancia, con la orquidometría de Prader.

Acuerdo entre observadores. En el presente trabajo, el acuerdo intraobservador para marcadores puberales masculinos fue excelente. Por su parte, el acuerdo interobservador fue excelente para la estadificación de Tanner, la orquidometría de Prader y Chipkevitch; en cambio, fue bueno para la orquidometría con Sotos.

Slora et al., ${ }^{18}$ evaluaron el acuerdo entre observadores en la estadificación de Tanner y orquidometría con Prader y obtuvieron resultados similares al presente trabajo. En su experiencia participaron 79 niños y 16 profesionales de la salud, en su mayoría pediatras, que recibieron capacitación en dos fases, una teórica y una práctica). En sus conclusiones, destacaron que, con la capacitación adecuada, los profesionales de atención primaria pueden obtener valores confiables de marcadores puberales masculinos. En época más reciente, un estudio de simulación con modelos 3D de testículos y escroto observó una gran variabilidad y pobre precisión en las mediciones intra e interobservadores, con una tendencia clara a sobrestimar el VT. ${ }^{19}$ Esta experiencia se realizó durante un encuentro de la Sociedad Británica de Endocrinología Pediátrica y Diabetes, con 215 participantes $(80 \%$ eran consultores o becarios en endocrinología pediátrica) y solo un $25 \%$ había recibido capacitación formal en medición testicular.

\section{Implicancias para la práctica}

Pese al uso generalizado del orquidómetro de Prader en la evaluación puberal, los estudios sobre concordancia en orquidometría clínica son escasos y difíciles de extrapolar a la población pediátrica sana, excepto la experiencia de Slora. ${ }^{19}$ Las pocas publicaciones disponibles corresponden a estudios realizados por urólogos $\mathrm{o}$ andrólogos, ${ }^{20,21} \mathrm{o}$ en pacientes con patología urogenital o endócrina previa. ${ }^{21-23}$

En el presente estudio se comparó la orquidometría de Prader con otras dos técnicas de medición testicular. El método de Chipkevitch podría ser intercambiable con el de Prader en la práctica diaria, dada su excelente concordancia en la medición del VT. En cambio, como era esperable, el método de Sotos mostró baja concordancia con el orquidómetro de Prader en la estimación del VT. Para evitar errores en la interpretación de los resultados, se debe tener en 
cuenta que la orquidometría de Sotos siempre obtiene menores valores de volumen testicular, en comparación con la de Prader y Chipkevitch, dado que no incluye los tejidos adyacentes en el cálculo del tamaño testicular.

La Sociedad Argentina de Pediatría promueve evaluar la precisión en las mediciones del crecimiento físico y mejorarla mediante programas de capacitación..$^{23}$ La orquidometría incluye diferentes métodos y requiere una capacitación adecuada para obtener una medición confiable..$^{5,12}$ Los autores consideran que dicha capacitación es necesaria, tanto en la formación médica básica como posbásica, para la evaluación, el diagnóstico, el tratamiento y el seguimiento del niño durante la pubertad y del niño con distintas alteraciones urogenitales.

\section{Limitaciones}

En el presente estudio no se realizó la comparación ecográfica. Es conocido que los métodos de Prader y Chipkevitch sobrestiman el volumen testicular en comparación a la ecografía. ${ }^{1,5,14-17}$ Sin embargo, publicaciones recientes llaman la atención sobre la gran variabilidad y la falta de consenso de la medición testicular por ecografía. ${ }^{24,25}$

Existe el riesgo de sesgo en la comparación de los tres métodos ante la imposibilidad de cegar las mediciones; el análisis estadístico fue cegado para mitigar este riesgo.

Los participantes provinieron de una población hospitalaria del sistema público de salud y fueron evaluados con un diseño transversal. Se recomienda cautela al extrapolar estos resultados. Se requieren estudios a gran escala sobre el desarrollo puberal de adolescentes argentinos, tanto en forma transversal como longitudinal.

\section{CONCLUSIONES}

Se comparó la concordancia de tres instrumentos de orquidometría clínica, con el orquidómetro de Prader como referencia. La orquidometría de Chipkevitch es sencilla, de bajo costo y mostró una excelente concordancia con la de Prader; por lo tanto, podrían intercambiarse en la atención clínica de niños y adolescentes. La orquidometría de Sotos, aunque de menor costo, tiene baja concordancia porque utiliza una escala de medición similar a la ecografía, y el tamaño testicular obtenido es siempre menor.

\section{REFERENCIAS}

1. Sotos J, Tokar N. Appraisal of testicular volumes: volumes matching ultrasound values referenced to stages of genital development. Int J Pediatr Endocrinol. 2017; 2017:7.

2. Chipkevitch E, Nishimura R, Tu D, Galea Rojas M. Clinical measurement of testicular volume in adolescents: comparison of the reliability of 5 methods. J Urol. 1996; 156(6):2050-3.

3. Society for Adolescent Health and Medicine; Arik V Marcell,
David L Bell, Alain Joffe, et al. The male genital examination: a position paper of the Society for Adolescent Health and Medicine. J Adolesc Health. 2012; 50(4):424-5.

4. Bell D, Breland D, Ott M. Adolescent and young adult male health: a review. Pediatrics. 2013; 132(3):535-46.

5. Dorn L, Dahl R, Woodward H, Biro F. Defining the boundaries of early adolescence: a user's guide to assessing pubertal status and pubertal timing in research with adolescents. Appl Dev Sci. 2006; 10(1):30-56.

6. Dorn L, Biro F. Puberty and its measurement: a decade review. J Res Adolesc. 2011; 21(1):189-95.

7. Giavarina D. Understanding Bland Altman analysis. Biochem Med (Zagreb). 2015; 25(2):141-51.

8. Lejarraga H, Heinrich J, Rodríguez A. Técnicas antropométricas. En: Comité Nacional de Crecimiento y desarrollo. Guía para la Evaluación del Crecimiento Físico. Ciudad Autónoma de Buenos Aires: Sociedad Argentina de Pediatría; 2013:19-24. [Acceso: 2 de enero de 2018]. Disponible en: http: / / www.sap.org.ar/docs/ publicaciones/libro_verde_sap_2013.pdf

9. Marshall W, Tanner J. Variations in the pattern of pubertal changes in boys. Arch Dis Child. 1970; 45(239):13-23.

10. Comité Nacional de Endocrinología. Enfoque práctico del manejo de la ginecomastia. Seis preguntas que debe responderse el pediatra ante un paciente con ginecomastia. Arch Argent Pediatr. 2011; 109(4):365-8.

11. Prader A. Delayed adolescence. Clin Endocrinol Metab. 1975; 4(1):143-55.

12. Prader A. Orchiometry. In Donald RA. Endocrine Disorders: a guide to diagnosis. New York: Marcel Dekker; 1984. Págs.33-6.

13. Chipkevitch E. Avaliação clínica da maturação sexual na adolescência. J Pediatr (Rio J). 2001; 77 (Suppl 2):S135-42.

14. Sotos J, Tokar N. Testicular volumes revisited: a proposal for a simple clinical method that can closely match the volumes obtained by ultrasound and its clinical application. Int J Pediatr Endocrinol. 2012; 2012(1):17.

15. Joustra S, van der Plas E, Goede J, Oostdijk W, et al. New reference charts for testicular volume in Dutch children and adolescents allow the calculation of standard deviation scores. Acta Paediatr. 2015; 104(6):e271-8.

16. Goede J, Hack W, Sijstermans K, van der Voort-Doedens $\mathrm{L}$, et al. Normative values for testicular volume measured by ultrasonography in a normal population from infancy to adolescence. Horm Res Paediatr. 2011; 76(1):56-64.

17. Tinggaard J, Grunnet Mieritz M, Sorensen K, Mouritsen A, et al. The physiology and timing of male puberty. Curr Opin Endocrinol Diabetes Obes. 2012; 19(3):197-203.

18. Slora E, Bocian A, Herman-Giddens M, Harris D, et al. Assessing Inter-rater reliability (IRR) of Tanner staging and orchidometer use with boys: A study from PROS. J Pediatr Endocrinol Metab. 2009; 22(4):291-9.

19. Elder C, Langley J, Stanton A, De SilvaS, et al. A simulation study assessing the accuracy and reliability of orchidometer estimation of testicular volume. Clin Endocrinol $(O x f) .2019$; 90(4):623-9.

20. Carlsen E, Andersen A, Buchreitz L, Jorgensen N, et al. Interobserver variation in the results of the clinical andrological examination including estimation of testicular size. Int $J$ Androl. 2000; 23(4):248-53.

21. Tatsunami S, Matsumiya K, Tsujimura A, Itoh N, et al. Inter/intra investigator variation in orchidometric measurements of testicular volume by ten investigators from five institutions. Asian J Androl. 2006; 8(3):373-8.

22. Rivkess S, Hall D, Boepple P, Crawford J. Accuracy and reproducibility of clinical measures of testicular volume. I Pediatr. 1987; 110(6):914-7.

23. Caino S. Estimación del error de medición. En Comité Nacional de Crecimiento y Desarrollo. Guía para la evaluación del crecimiento físico. Ciudad Autónoma de Buenos Aires: Sociedad Argentina de Pediatría; 2013:25-6. [Acceso: 2 de enero de 2018]. Disponible en: http: / / www. sap.org.ar/docs/publicaciones/libro_verde_sap_2013.pdf

24. Welliver C, Cardona-Grau D, Elebyjian L, Feustel PJ, Kogan B. Surprising interobserver and intraobserver variability in pediatric testicular ultrasound volumes. J Pediatr Urol. 2019; 15(4):386.e1-6.

25. Sorokin I, Welliver C, Elebyjian L, Feustel P, McCullough A. Interinstitutional variability in testicular volumes and varicocele presence by ultrasound: surprising discrepancies and implications for clinical decision making. Urology. 2015; 85(5):1079-84. 